\title{
Validation of Shielding Analysis Capability of SuperMC with SINBAD
}

\author{
Chaobin Chen, Qi Yang, Bin Wu, Yuncheng Han, Jing Song*, FDS Team \\ Key Laboratory of Neutronics and Radiation Safety, Institute of Nuclear Energy Safety Technology, Chinese Academy of Sciences, Hefei, \\ Anhui, 230031, China \\ * Corresponding Author, E-mail: jing.song@fds.org.cn
}

\begin{abstract}
The shielding analysis capability of SuperMC was validated with the Shielding Integral Benchmark Archive Database (SINBAD). The SINBAD was compiled by RSICC and NEA, it includes numerous benchmark experiments performed with the D-T fusion neutron source facilities of OKTAVIAN, FNS, IPPE, etc. The results from SuperMC simulation were compared with experimental data and MCNP results. Very good agreement with deviation lower than $1 \%$ was achieved and it suggests that SuperMC is reliable in shielding calculation.
\end{abstract}

KEYWORDS: Monte Carlo, SuperMC, SINBAD, Validation

\section{Introduction}

SuperMC is a general purpose, easy-to-use Monte Carlo software for modeling and simulation of nuclear facilities. The latest version of SuperMC can perform the simulation of neutron, photon and coupled neutron and photon transport, and also equipped with the function of automatic modeling and visualization ${ }^{[1-2]}$.

For neutron transport, elastic scattering, inelastic scattering, and various absorption reaction processes includes ( $n, x n)$, (n, f), (n, $\gamma$ ), etc., with neutron energies from $0 \mathrm{MeV}$ to 150 $\mathrm{MeV}$, are considered. For photons, the code accounts for Compton scattering, coherent scattering, possibility of fluorescent emission after photoelectric absorption, electron-positron pair production and photonuclear processes with photon energies from $1 \mathrm{keV}$ to $100 \mathrm{GeV}$. SuperMC uses high-resolution pointwise cross-section databases created from ENDF/B-6 format evaluated nuclear data libraries.

SINBAD is a compilation of 77 fission and fusion shielding experiments, most of which have been modeled with Monte Carlo (McBEND, TRIPOLI, MCNP) or deterministic codes (ANISN and DOT3.5). Shielding analysis is a typical application of SuperMC, and the primary objective for the radiation shielding validation is to assess the impact of SuperMC methodology and its neutron and photon data libraries on attenuation factors and dose rates. D-T fusion neutron source benchmark cases were selected to validate the shielding analysis capability of SuperMC.

\section{Validation Method}

SuperMC has been extensively tested and validated inhouse over the years with benchmark cases from criticality safety experiments ${ }^{[3]}$ and some typical radiation shielding cases, like ITER benchmark problems ${ }^{[4]}$. In this work, SuperMC transport functions were validated against benchmark experiments from three D-T neutron source facilities compiled in SINBAD. The problems with time-offlight spectra were chosen to test several features of the code, such as neutron and photon transport, secondary photon production, coupling of the neutron-photon transport, point detector, boundary crossing detector or surface detector, and angular flux. In the next work, dose rates from neutrons and photons would be concerned.

A brief description of selected experiments was given below, and the calculation results were presented in the next section.

\section{Osaka experiments}

Neutron leakage spectra from different diameters of iron, aluminum, silicon, nickel and tungsten sphere were measured between $10 \mathrm{keV}$ and $15 \mathrm{MeV}$ by time-of-flight techniques. Some measurements were carried out for two energy regions, the high energy region for high energy spectrum and the low energy region for low energy spectrum, such as iron and nickel sphere experiments. In some cases, gamma-ray leakage spectra were measured, such as aluminum, silicon and tungsten sphere experiments.

\section{FNS experiments}

Materials including graphite, oxygen, vanadium, iron, tungsten, lead, etc., had been performed on the FNS facility. The neutron spectra emerging from slabs of material of varying thickness were measured at five different angles. The slabs were placed at $20 \mathrm{~cm}$ distance from a $14 \mathrm{MeV}$ DT neutron source.

\section{IPPE experiments}

Time-of-flight measurements of neutron leakage spectra from iron, nickel, vanadium, bismuth, uranium and thorium spheres with central $14 \mathrm{MeV}$ neutron sources and from uranium and thorium sphere with ${ }^{252} \mathrm{Cf}$ sources were performed. 


\section{Results}

Benchmark experiments were modeled by modeling function of SuperMC, and all the problems were run on single CPU computers with ENDF/B-VII.1 neutron cross section library and ENDF/B-VI.8 photon cross section library. Integrated results over energy were dicussed and typical graphs were presented in this section.

\section{Osaka benchmarks}

SuperMC was used to simulate Osaka benmarks to get the neutron and photon leakage spectra from $\mathrm{Al}, \mathrm{Si}, \mathrm{Fe}, \mathrm{Ni}$ and $\mathrm{W}$ spheres. The experiments were modeled with a simple spherical geometry, and two isotropic point sources, a neutron source and a photon source, located in a spherical air cavity at the center of the spheres. The results were compared with measured data and calculated values from MCNP. The integrated leakage fluxes over energy were in good greement with experimental data and within $0.1 \%$ with MCNP results. Leakage spectrums from $50 \mathrm{~cm}$ iron sphere and $16 \mathrm{~cm}$ nickel sphere are shown in Fig. 1.
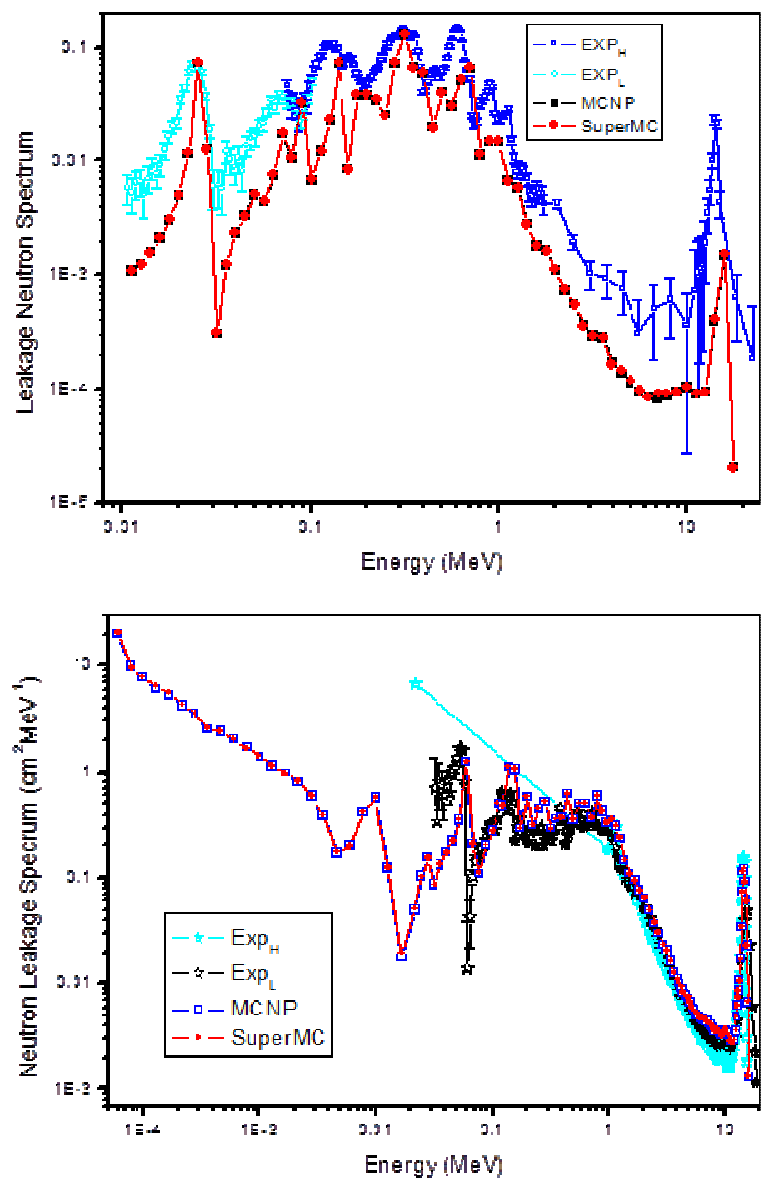

Fig.1 Comparison of neutron leakage currents of Osaka benchmark experiments (Up: Iron, Down: Nickel)

\section{FNS benchmarks}

The FNS time-of-flight benchmark specifications are given on the IAEA web site ${ }^{[5]}$ and in SINBAD. Experiments with materials of $\mathrm{C}, \mathrm{O}, \mathrm{V}, \mathrm{W}$ were simulated using SuperMC, with neutron source spectrum taken from the reference ${ }^{[6]}$ and an isotropic neutron distribution assumed. The differences of point integrated flux over energy are within $1 \%$ compared with calculated results by MCNP and the point flux spectra of $20 \mathrm{~cm}$ oxygen slab in two angles are shown in Fig. 2.
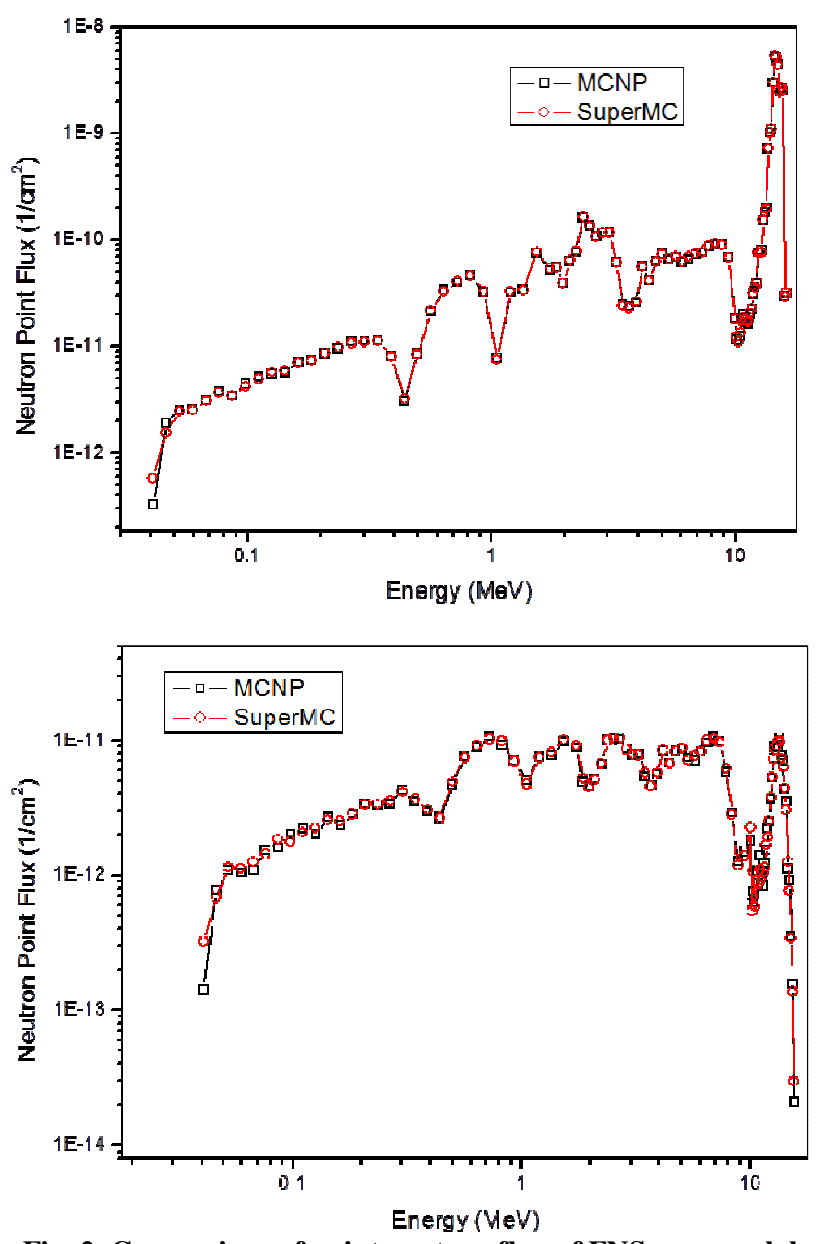

Fig. 2. Comparison of point neutron flux of FNS oxygen slab benchmark experiments (Up: 0 degree, Down: 66.8 degree)

\section{IPPE benchmarks}

$\mathrm{V}$ and Th spheres were simulated using SuperMC with $14 \mathrm{MeV}$ neutron source. The differences of point integrated flux over energy were about $0.1 \%$ compared with calculated results by MCNP and the point flux spectra of thorium sphere are shown in Fig. 3. 


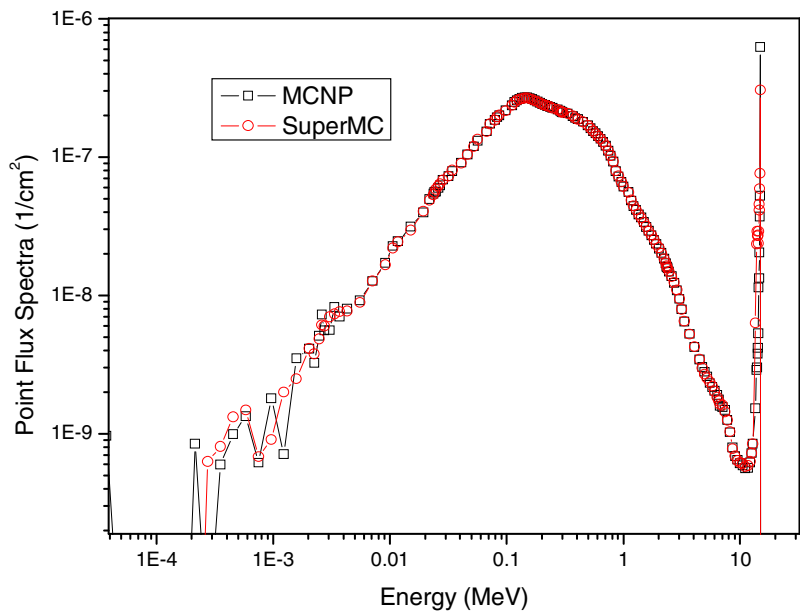

Fig. 3. Comparison of point neutron flux of Th sphere experiments with $14 \mathrm{MeV}$ neutron source

\section{Conclusion}

SuperMC had been verified with D-T fusion neutron source benchmark cases from SINBAD, and excellent agreement has been obtained between SuperMC and MCNP. The correctness and reliability of SuperMC transport function and high accessibility of modeling function were fully validated.

\section{Acknowledgment}

The work was supported by the Strategic Priority Research Program of Chinese Academy of Sciences (No. XDA03040000), the National Special Program for ITER (No. 2014GB112001), the National Natural Science Foundation of China (No.11305205).

\section{References}

1) WU YC, SONG J, ZHENG HQ, et al, CAD-based Monte Carlo Program for Integrated Simulation of Nuclear System SuperMC, Annuals of Nuclear Energy, DOI: 10.1016/j.anucene.2014.08.058.

2) WU YC, FDS TEAM, CAD-based Interface Programs for Fusion Neutron Transport Simulation, Fusion Engineering and Design, 84, 1987 (2009).

3) Binhang Zhang, Criticality Validation of SuperMC with ICSBEP, Annals of Nuclear Energy, 87, 494-499 (2016).

4) Jing Song, Benchmarking of CAD-based SuperMC with ITER benchmark model, Fusion Engineering and Design, 89, 2499-2503 (2014).

5) www-nds.iaea.or.at/fendl2/validation/benchmarks/ jaerim94014/fns-tof

6) M.Z. Youssef, Verification of ITER shielding capability and FENDL data benchmarking through analysis of bulk shielding experiment on large SS316/water assembly bombarded with $14 \mathrm{MeV}$ neutrons, Fusion Engineering and Design, 42, 235-245 (1998). 